\title{
Examining the Effectiveness of Fingerspelling in Improving the Vocabulary and Literacy Skills of Deaf Students
}

\author{
Hadeel Alawad, Millicent Musyoka* \\ Lamar University, Beaumont, USA \\ Email: halawad@lamar.edu,*mmusyoka@lamar.edu
}

How to cite this paper: Alawad, H., \& Musyoka, M. (2018). Examining the Effectiveness of Fingerspelling in Improving the Vocabulary and Literacy Skills of Deaf Students. Creative Education, 9, 456-468. https://doi.org/10.4236/ce.2018.93032

Received: January 26, 2018

Accepted: March 24, 2018

Published: March 27, 2018

Copyright ( 2018 by authors and Scientific Research Publishing Inc. This work is licensed under the Creative Commons Attribution International License (CC BY 4.0).

http://creativecommons.org/licenses/by/4.0/

\section{cc (i) Open Access}

\begin{abstract}
This article provides a narrative review of journal articles on the use of fingerspelling to support the vocabulary and literacy development of deaf students. The role of fingerspelling in decoding written English and supporting vocabulary development has become an increasingly relevant topic in the bilingual education of deaf students. Search limiters included written English, full-text articles that were published in peer-reviewed journals after 2005. A total of eleven articles were reviewed. The findings from the review indicated that the use of fingerspelling could support vocabulary and literacy development among bilingual deaf students. The current review discusses the findings and offers recommendations for future research.
\end{abstract}

\section{Keywords}

Deaf, Fingerspelling, Vocabulary, Literacy

\section{Introduction}

The academic success of deaf students relies on their ability to read and comprehend the meaning of a text. Thus, the development of literacy skills is considered a critical factor in deaf students' academic success. Difficulty in reading and writing among deaf students adversely affects their learning processes, indicating the importance of literacy skill development for deaf students (Alawad, 2011; Fayad, 2004; Williams, 2012). According to Sarchet, Marschark, Borgna, Convertino, Sapere, and Dirmyer (2014), deaf children usually score much lower on vocabulary tests than hearing children. Similarly, Goldin-Meadow and Mayberry (2001) indicated that "most profoundly deaf read poorly" (p. 222). Since the primary objective of education for deaf students is to teach them how to read 
and write in English, the improvement of English literacy skills among deaf students is considered fundamental. As stated by Berent (2001), "Sound knowledge of English is a critical factor in students' academic success and the attainment of gainful employment" (p. 1). Several factors may affect deaf students' ability to acquire English literacy skills. According to Alawad (2011), deaf students indicate below-average reading and writing skills, perhaps due to other factors related to the education environment, such as teaching methods, unsuitable curricula, and a lack of skilled teachers. Furthermore, visual communication strategies are regarded as significant sources for deaf students' attainment of knowledge (Alnagi, 2010).

Numerous studies have noted the influence of American Sign Language (ASL) in deaf education. Prinz and Strong's $(1997,1998)$ studies found an affirmative link between the ASL proficiency of deaf children and their skills in English literacy, irrespective of the children's ages or intelligence quotients (IQs). In particular, several articles and studies have discussed the importance of fingerspelling and illustrated that the use of fingerspelling promotes the comprehension and retention of academic knowledge (Baker, 2010). Fingerspelling can be essentially defined as the process of representing vocabulary symbols through the use of hand shapes and movements to spell alphabetic characters (Baker, 2010). Fingerspelling is regarded as a fundamental part of sign language (HaptonstallNykaza \& Schick, 2007). According to Grushkin (1998), deaf Americans tend to merge fingerspelling with language structure, and they usually use fingerspelling for names, pronouns, verb, nouns, and articles, such as the and an. The integration of fingerspelling and signs via various fingerspelling strategies, such as abbreviations, lexicalization processes, and signed-fingerspelling compounds, may help increase the ASL lexicon and develop literacy skills among deaf children (Allen, 2015; (Baker, 2010).

There is a strong relationship between fingerspelling proficiency and the development of literacy skills since deaf children rely on fingerspelling as a visual tool to decode and recollect words' symbols to write them (Grushkin, 1998; Haptonstall-Nykaza \& Schick, 2007). As stated by Haptonstall-Nykaza and Schick (2007), fingerspelling is an important key to deaf children's achievement of academic success in reading.

Proponents of many bilingual strategies have endorsed the use of various fingerspelling methods, such as the chaining strategy, in deaf classrooms to develop literacy skills for deaf students. Padden and Ramsey (2000) explained that teachers use a method called chaining to help deaf children connect English words and signs through fingerspelling. In this strategy, the teacher fingerspells a vocabulary word, then points to the print word, and finally signs the word. For example, "When teaching the word, tornado, a teacher might choose one of the following sequences: point to the word tornado written on the board; fingerspell T-O-R-N-A-D-O; and sign TORNADO or fingerspell TORNADO; sign TORNADO; and write tornado on the board" (Baker, 2010: p. 4). According to 
Humphries and MacDougall (1999), the combination of fingerspelling, signs, and print words in the use of a chaining strategy strongly indicates the existence of an achievable and natural contiguity among ASL and English students in the classroom. Furthermore, Quinto-Pozos and Reynolds (2012) reported that the use of a chaining strategy and a connecting-explaining technique to connect deaf students' cultural backgrounds to written text promotes communication via ASL. Gaston (2009) also suggested that utilizing a fingerspelling stories strategy may help deaf students acquire new English words. In sum, these research studies have indicated the significance of adopting fingerspelling bilingual strategies in teaching vocabulary and literacy skills to deaf students. They also have suggested the use of fingerspelling bilingual strategies, such as a chaining strategy, a connecting-explaining technique, or a fingerspelling stories strategy, to assist deaf students in connecting fingerspelling with printed words.

\section{Identifying the Research Questions}

The following research questions guided the present review:

1) How does fingerspelling impact vocabulary development?

2) How does fingerspelling impact literacy development?

3) What guidelines for educational practitioners can be derived from this body of knowledge?

\section{Method}

An electronic search was conducted using Lamar University databases, including the ERIC, PsycINFO, and Humanities (full text) databases. Various journals, including Journal of Deaf Studies and Deaf Education, American Annals of the Deaf, Sign Language Studies, Journal of Developmental \& Physical Disabilities, and Journal of Language and Literature were also consulted. Google databases for the years 2005 to 2015 were also referenced. The search included the following terms: "deaf and fingerspelling," "fingerspelling strategies," "chaining strategy," "fingerspelling and vocabulary," and "fingerspelling and literacy." The criteria that guided the search and selection of the articles reviewed included (a) studies done with deaf and hard of hearing students as participants, (b) articles restricted to peer-reviewed journals, (c) accessible full-text articles, (d) articles published between 2005 and 2016 and (e) articles published in English. The selection was not limited to studies conducted in the United States.

In sum, the search returned 229 articles. Of these, 101 articles were excluded upon the title review and 94 articles were rejected for not meeting the critical search, resulting in a total of 34 articles considered relevant or possibly relevant. Of these, 23 articles were excluded because the authors couldn't access full articles of the studies for review. Thus, a total of 11 articles were ultimately reviewed. These studies focused on deaf children between elementary to adult age. All 11 research studies were published after 2005. Figure 1 present the process of selecting the articles reviewed. 


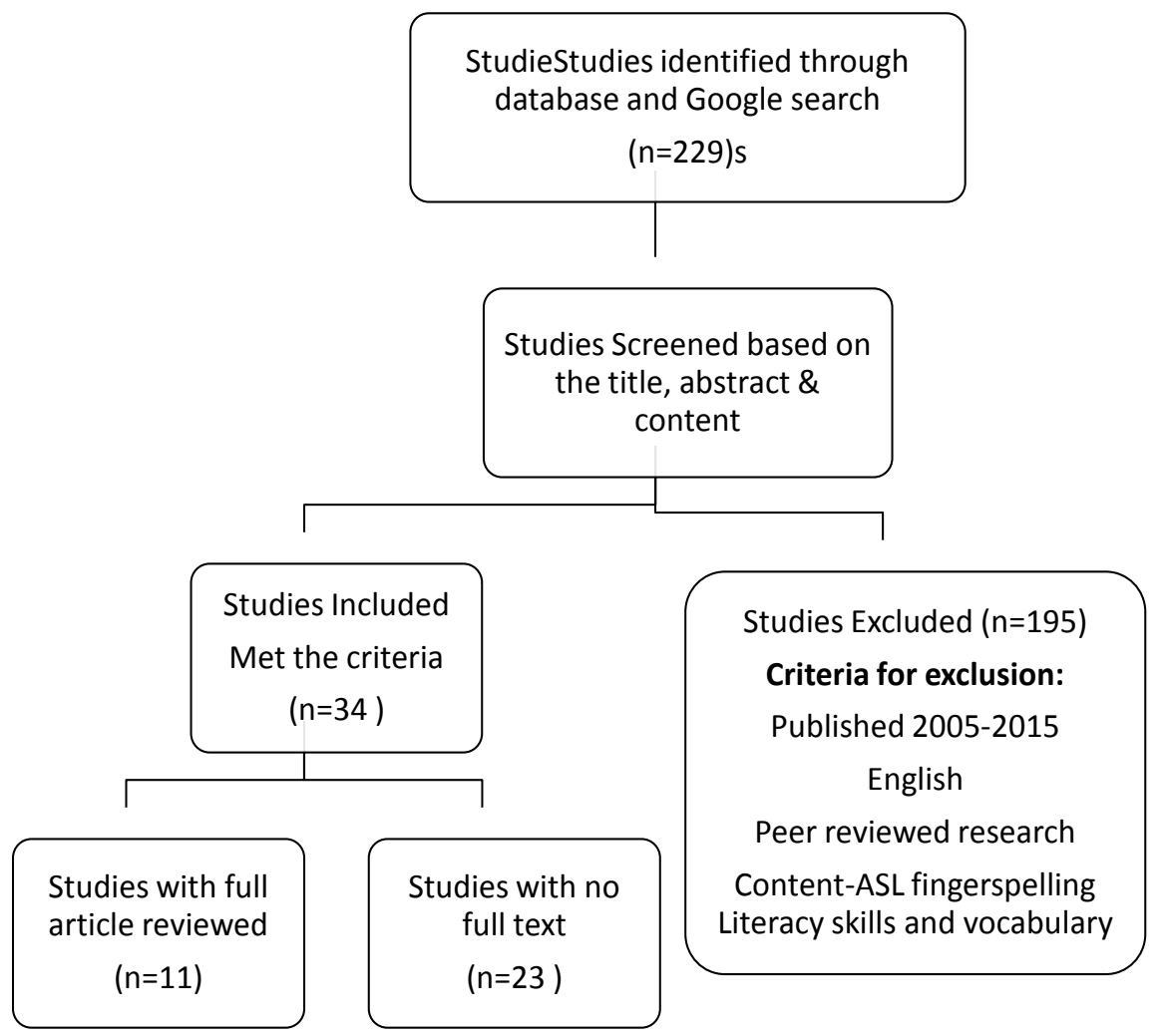

Figure 1. Articles selection process.

Based on the selection criteria process set in this study the journal articles reviewed in this paper are presented in Table 1.

\section{Results}

\subsection{Fingerspelling and Vocabulary Development}

Vocabulary development refers to the process by which a child develops vocabulary. Vocabulary includes two kinds of words: receptive words and expressive words. Receptive vocabulary includes words that a child comprehends when he/she reads them, while expressive vocabulary consists of the words that a child uses when he/she expresses him/herself independently through speaking or signing. Williams (2012) indicates that developing a large vocabulary bank is critical because students are required to read and comprehend different types of books in school. He asserts that "vocabulary knowledge is strongly associated with reading achievement and becomes increasingly predictive of overall reading proficiency as children progress through the elementary grades" (p. 501). Trussell and Easterbrooks (2014) argue that early word acquisition has a positive impact on the development of a child's reading and writing skills and that there is a strong correlation between vocabulary development and literacy.

The importance of American Sign Language fingerspelling in the development of vocabulary skills for deaf students has been shown in the literature. Puente, Alvarado, and Herrera (2006) investigated the role of sign language and 
Table 1. Description of the studies reviewed.

\begin{tabular}{|c|c|c|c|}
\hline Author & Study design & Participants & Findings \\
\hline $\begin{array}{l}\text { Puente, Alvarado } \\
\text { \& Herrera (2006) }\end{array}$ & Experimental & $\begin{array}{l}n=26 \\
\text { Ages } 7-15\end{array}$ & $\begin{array}{l}\text { Strong link between orthographic c skills and use of } \\
\text { fingerspelling skills } \\
\text { Fingerspelling and visual coding of vocabularies }\end{array}$ \\
\hline $\begin{array}{l}\text { Haptonstall-Nykaza } \\
\text { \& Schick (2007) }\end{array}$ & $\begin{array}{l}\text { Pre/post, Quasi } \\
\text { experimental design }\end{array}$ & $\begin{array}{l}\mathrm{n}=21 \\
\text { Ages } 4-14 \mathrm{yrs}\end{array}$ & Word identity and English writing skills improved \\
\hline Hile (2009) & $\begin{array}{l}\text { Quantitative } \\
\text { Experimental research }\end{array}$ & $\begin{array}{l}\mathrm{n}=55 \\
\text { Ages } 5-9 \mathrm{yrs}\end{array}$ & $\begin{array}{l}\text { Age and family characteristics influence the effect of } \\
\text { fingerspelling on vocabulary } \\
\text { R/shp between fingerspelling and literacy skills noted. }\end{array}$ \\
\hline Gaston (2009) & Qualitative research & $\begin{array}{l}\mathrm{N}=5 \\
\text { Ages } 6-9\end{array}$ & $\begin{array}{l}\text { Fingerspelled stories assisted students in learning new } \\
\text { vocabularies }\end{array}$ \\
\hline Stone, et.al. (2015) & Quantitative research & $\begin{array}{l}\mathrm{N}=32 \\
\text { Ages } 19-44 \text { yrs }\end{array}$ & $\begin{array}{l}\mathrm{R} / \text { ship between fingerspelling, sign language and } \\
\text { orthographic decoding }\end{array}$ \\
\hline Schwartz (2011) & Qualitative research & $\begin{array}{l}N=10 \\
\text { Ages } 5-10\end{array}$ & Phonological awareness and fingerspelling \\
\hline $\begin{array}{l}\text { Staden \& } \\
\text { Roux (2010) }\end{array}$ & $\begin{array}{l}\text { Quasi experimental } \\
\text { design }\end{array}$ & $\begin{array}{l}N=64 \\
\text { Ages } 9-10 \mathrm{yrs}\end{array}$ & $\begin{array}{l}\text { Fingerspelling, visual picture and print language enhance } \\
\text { literacy skills development }\end{array}$ \\
\hline $\begin{array}{l}\text { Quinto-Pozos } \\
\text { \& Reynolds (2012) }\end{array}$ & Qualitative research & $\begin{array}{l}\mathrm{N}=2 \text { Deaf, native } \\
\text { signers of ASL }\end{array}$ & $\begin{array}{l}\text { Use of chaining fingerspelling technique support vocabulary } \\
\text { development and print word recognition. }\end{array}$ \\
\hline $\begin{array}{l}\text { Emmorey \& } \\
\text { Petrich (2012) }\end{array}$ & $\begin{array}{l}2 \text { Experimental } \\
\text { research }\end{array}$ & $\begin{array}{l}\text { Experiment } 1 \\
\mathrm{~N}=52 \text { deaf signers and } \\
32 \text { hearing nonsigners } \\
\text { Experiment } 2 \\
36 \text { deaf signers }\end{array}$ & Mouthing and fingerspelling promote phonological awareness. \\
\hline $\begin{array}{l}\text { William, Darcy } \\
\text { \& Newman (2015) }\end{array}$ & Experimental & $\begin{array}{l}\mathrm{N}=31 \text { Deaf high school } \\
\mathrm{N}=24 \text { university students }\end{array}$ & Fingerspelling supports print orthography \\
\hline Roos (2013) & Qualitative & $\begin{array}{l}\mathrm{N}=6 \\
\text { Age: Preschool }\end{array}$ & Fingerspelling in play support decoding of written text. \\
\hline
\end{tabular}

fingerspelling in improving the literacy skills of deaf children and adolescents. Their experimental study sample included 26 deaf participants ages seven through 15 who used Chilean Sign Language as their primary language. The researchers examined the participants' abilities in (a) identifying Chilean signs and fingerspelling vocabularies, (b) matching fingerspelling vocabularies with commercial slogans, and (c) decoding fingerspelling vocabularies and mapping individual vocabularies into written form. The study's results confirmed the presence of a strong link between orthographic skills and the use of fingerspelling and sign language. Findings revealed that older deaf students recognized signs and fingerspelling better than the younger deaf students did. Moreover, deaf adolescents performed better in sign language recognition than in recognition of fingerspelling. Fingerspelling serves as a visual code for word recognition. The results strongly suggest that fingerspelling can simplify the internal representation of vocabularies.

Haptonstall-Nykaza and Schick (2007) conducted a Pre/post, Quasi experimental design to examine whether training methods that used lexicalized fin- 
gerspelling, signs, and English vocabulary to teach deaf students new English words would enhance their ability to learn fingerspelling and the English spelling system. The participants were 21 deaf students ages four to 14 years old. Nine had deaf parents, and 12 had hearing parents. The participants were divided into two groups. The first group was taught the new English vocabulary using American Sign Language (ASL). The other group was taught using fingerspelling, with the teacher making associations between the English vocabulary, the signs, and the lexicalized fingerspelling. The researchers found that when the training process included lexicalized fingerspelled words, deaf students were better able to understand and fingerspell the word and to write the printed English word. In addition, the deaf students who had deaf parents acquired more vocabulary in both sign and fingerspelling conditions compared with the deaf students of hearing parents. The researchers asserted that the lexicalized fingerspelling strategy made explicit the relationship between the signs and the English print. Moreover, this study found that ASL has naturally established a system that explains the relationship between signs and written English.

Along the same lines as these previous studies, Hile (2009) examined the relationship between deaf children's ability to learn fingerspelled vocabulary and their literacy skills. This quantitative experimental research aimed to study deaf children's ability to quickly map familiar and newly learned fingerspelled vocabulary during a coaching task. The study sample included 55 deaf children ages five to nine years old who had either hearing or deaf parents. The learning process was evaluated in five areas: imitation, matching, production, lexical identification, and writing. The study's results indicated that older deaf children learned more fingerspelling vocabulary than younger deaf children. Regardless of age, deaf children from deaf parents learned more vocabulary than deaf children from hearing parents. The results of the study suggest that there is a substantial relationship between deaf children's ability to learn new vocabulary via fingerspelling and their literacy potential. The study pointed to the significant role that fingerspelling plays in literacy and bilingual language development.

Similarly, Gaston (2009) conducted a qualitative study to ascertain how deaf and hard-of-hearing students evolved in terms of spelling English vocabulary through the provision of lessons that used a visual strategy. Five deaf students were selected as a sample. The researchers used fingerspelled stories related to different concepts to accelerate learning in relation to the adoption of new vocabulary. To enrich the goals of each lesson, an evaluation plan was applied to allow the students to attain optimal improvements. The data collection methods used in the evaluation plan was tests, artifact collection, field notes and rubrics. This evaluation plan was capable of identifying the effectiveness of projected programs on learning lessons, and it also aided student development through the inclusion of feedback on students' growth prospects. Moreover, from analyzing the evaluation data, the fingerspelled stories were able to create useful results for 
all students. The fingerspelling method assisted these students in learning new vocabulary; thus, the students experienced greater exposure to ASL through learning English vocabulary.

In line with this hypothesis, Stone, Kartheiser, Hauser, Petitto, and Allen (2015) used a quantitative approach to explore the link between several independent variables (the ages at which deaf college students were first exposed to ASL, their current fluency in ASL, and their fingerspelling skills) and reading potential. The study's sample included 32 deaf individuals, whose age arrange between 19 to 44 years old from Gallaudet University and the greater Washington, D.C. metropolitan area who had achieved reading fluency and a working proficiency in fingerspelling skills. The results showed that promoting the relationships among fingerspelling, sign language, and orthographic decoding may facilitate the development of English reading skills.

\subsection{Fingerspelling and Literacy Development}

Literacy development plays a fundamental role in students' educational success. As such, delays in the development of early literacy skills can lead to academic failure. The term 'emergent literacy' refers to the acquisition of oral language skills (e.g., expressive and receptive vocabularies and word knowledge) and decoding skills (e.g., letter naming, phonological awareness, and print knowledge) (Kim, Im, \& Kwon, 2015). Educators in the field of deaf education argue that early exposure to language and literacy is crucial for deaf children (Golos \& Moses, 2013). Padden and Ramsey (2000) stated that the knowledge of American Sign Language and fingerspelling is positively associated with reading performance. Fingerspelling creates a linguistic correlation between printed words and syntax (Baker, 2010). Puente et al. (2006) suggested that fingerspelling "can act as a complementary means of decoding in reading processes, and perhaps aid in the development of phonemic awareness in signing deaf children, though it is a manual system for representing the alphabetic rather than phonemic units of language" (p. 300).

Many studies have examined the relationship between fingerspelling and phonological awareness and how fingerspelling can support phonological awareness. In a study of 10 deaf and hard-of-hearing participants (four boys and six girls) ages five to 10 years old (kindergarten through fourth grade), Schwartz (2011) conducted a qualitative research to investigate the relationship between phonological awareness and fingerspelling by giving participants phonological awareness tasks using both speech and fingerspelling conditions. The researcher's aim was to explore the internal vocabulary awareness of deaf and hard-ofhearing students and understand their capacity to adapt their phonemic purview of language via fingerspelling. The study found that deaf and hard-of-hearing children displayed phonological awareness in fingerspelling conditions for all tasks.

Staden and Roux (2010) conducted a study to examine the hypothesis that 
deaf children face considerable challenges in both reading and writing due to their limitations in phonological coding. The researchers identified bilingual and bicultural programs that encouraged the development of sign language and visual coding skills among deaf children. The approaches of these programs were designed to enhance deaf children's written skills by helping them to make phonological connections to written words. A quasi-experimental design was used to validate the research hypothesis. The study sample included deaf children with spelling problems from a rural South African residential school for the deaf. The children were divided into two groups: 32 students were placed in the treatment group (mean age $=119.19$ months) and 32 students were placed in the control group (mean age $=117$ months). The researcher developed a spelling mastery program that contained several computer-based exercises and worksheets to teach deaf children to use fingerspell coding. The results show that the children in the treatment group were able to increase their spelling capabilities. The researchers state that the utilization of the spelling mastery program, which incorporates fingerspell coding, visual pictures, and print language mapping, was especially useful in improving the spelling skills of the children in the treatment group. This study highlighted the importance of employing fingerspell coding and visual imaging techniques in teaching and enhancing literacy development among deaf students.

Quinto-Pozos and Reynolds (2012) investigated the manner through which native deaf signers use contextualization strategies to write in English and correctly match words with meanings. The qualitative study recommended two strategies-"chaining" and "connecting-explaining"-for communicating in ASL using the contextualization technique. The chaining strategy is a visual technique that integrates fingerspelling, printed words, and sign language into classroom instruction. In this strategy, a teacher fingerspells a vocabulary word, then points to the print word, and finally signs the word. Two deaf respondents presented this text to audiences in multiple locations. To maintain control over the content from one performance to another, the single text capitation method was used. In presenting the text, the deaf presenters applied linguistic and other communication strategies that assisted them in coping with the presentation settings. The study revealed that both the chaining and connecting-explaining strategies are used in parsing the ASL text; however, the connecting-explaining technique was largely related to the chaining strategy. The study was able to contribute to and develop a clear perspective on ASL techniques and explained the implications of these techniques for professionals, including teachers of deaf children, sign language interpreters, linguists, and sign language mentors.

Likewise, Emmorey and Petrich (2012) conducted an experimental study to ascertain the relationship between fingerspelling and print in orthographic structures. The authors explored similar approaches for reading printed and fingerspelled words by conducting two lexical decision experiments. The first experiment tested orthographical and phonological syllable segregation methods 
among groups of 52 deaf signers and 32 hearing non-signers. These were tested through correlation analysis. All of the deaf signers in the experiment were capable of using ASL; however, the hearing respondents did not have any ASL knowledge. This experiment showed that both deaf and hearing readers can perform well if written words are segregated by orthographical syllables rather than by phonological syllables. The study suggested that the use of orthographic syllables was more suitable for deaf readers. However, segmentation strategies were not able to differentiate between the levels of reading ability within the hearing group. In its second experiment, the study sought to determine whether printed words based on orthographic preferences could be transferred to fingerspelled words. To answer this question, 36 deaf signers (mean age $=28$ years) who had participated in the first experiment were selected. The results of the second experiment suggested that the deaf students performed better in the limited segment related to phonological syllables. Correspondingly, the study suggested that mouthing of the English words should accompany fingerspelling to promote better performance of phonological techniques since fingerspelling is connected to both comprehension and vocabulary skills. The results of the study showed that neither the print technique nor the fingerspelling technique is rooted in strategies of shared segmentation for adult deaf readers. The study recommended constructing a powerful representation of English to facilitate and enhance the fingerspelling technique and improve readers' English vocabulary skills.

Along the same lines, Williams, Darcy, and Newman (2015) conducted an experimental study to determine the influences of orthography and spoken phonology on the recognition of visual words in print and fingerspelling. The researchers anticipated that the particular practice of orthographically written words would be able to increase the priming abilities of deaf students. The study determined that fingerspelling identification could give rise to the same types of priming effects in print. Thus, two different experiments were conducted to understand the impacts of priming. Thirty-one deaf students from the Indiana High School for the Deaf participated in the first experiment. The results suggested that print orthography priming has the same effect on deaf readers as listening literature. Moreover, this impact is not similar to that of English phonological priming. The second experiment was conducted to study fingerspelling priming among both hearing and deaf signers. The experiment sample included the deaf student group that had participated in the first experiment as well as 24 students from Indiana University. It was observed that deaf signers experience orthographically comparable effects in reaction to both print and fingerspelling. Thus, the study concluded that ASL students interpret fingerspelling accurately through direct contact with the orthographical illustrations of the words. In sum, both of these experiments suggested that deaf students implement similar mechanisms to process orthography and fingerspelling.

In 2013, Roos conducted a qualitative study to explore the various roles that 
fingerspelling plays in the development of writing and reading skills of deaf children prior to and during their first school years. The study included six deaf children age range between three and six years; who were enrolled in preschool classes in a Swedish town. The study found that the deaf children used fingerspelling in play as a sign of cultural identity; in addition, they used fingerspelling as a way to reminisce about or decode written text.

\section{Discussion}

This review of existing literature makes clear that there is a positive relationship between fingerspelling and children's improved English spelling and learning of new vocabulary (Gaston, 2009; Hile, 2009; Haptonstall-Nykaza \& Schick, 2007; Puente et al., 2006; Stone et al., 2015). In addition, there is substantial evidence that a combination of signs, fingerspelling, printed words, and bilingual fingerspelling strategies, such as the lexicalized fingerspelling strategy, chaining, fingerspelling stories, and the connecting-explaining strategy, can help deaf students recognize and learn new vocabulary and develop their reading and writing skills (Gaston, 2009; Haptonstall-Nykaza \& Schick, 2007; Stone et al.,2015). In addition, there is strong evidence that early exposure to ASL and fingerspelling facilitates the development of English literacy skills (Puente et al., 2006). Furthermore, there is a strong relationship between the development of visual coding based on fingerspelling and the improvement of deaf children's literacy skills (Emmorey \& Petrich, 2012; Roos, 2013; Schwartz, 2011; Staden \& Rouy, 2010; Williams et al., 2015). Moreover, several studies suggest that deaf students use fingerspelling as a visual way to reminisce, decode, read, and write English words, as well as a way to express their cultural identity (Emmorey \& Petrich, 2012; Haptonstall-Nykaza \& Schick, 2007; Roos, 2013; Schwartz, 2011; Staden \& Rouy, 2010; William et al., 2015).

\section{Conclusion}

The purpose of this review was to examine the research from the past ten years on fingerspelling and its relationship to vocabulary and literacy development for deaf students. It is clear from the reviewed research that there is a strong link between fingerspelling and the development of vocabulary and literacy skills among deaf students. Thus, it is recommended that educational programs for deaf students adapt ASL/fingerspelling/English bilingual strategies to assist deaf students in developing their vocabulary and literacy skills. The research also suggests that all teachers of deaf students need to be proficient in both ASL and the English language and need to be trained in using the bilingual fingerspelling strategy to reinforce the vocabulary, reading, and writing development of deaf students. Moreover, it is recommended that teachers at schools for the deaf be conscious and knowledgeable about the differences in the vocabulary and literacy development of hearing versus deaf students. Teachers need to understand that deaf students depend on fingerspelling as a visual means to decode words 
and work to employ fingerspelling in their classroom instruction. Since early language acquisition is critical in literacy development, parents and early intervention professionals need to be familiar on how they can introduce and use fingerspelling strategies to support early language acquisition and development. Finally, there is an urgent need to conduct future research on how teachers, parents and early intervention professional use fingerspelling strategies (e.g., chaining, sandwiching) in developing vocabulary and literacy skills. More studies are needed to examine its effectiveness in improving vocabulary and literacy skills among deaf learners.

\section{Limitations}

Several limitations of this review were identified. In terms of inclusion criteria, the articles were limited to full-text articles written in English and published in peer-reviewed journals after 2005. The scope of the review was limited to studies focusing on ASL fingerspelling and English literacy skills.

\section{References}

Alawad, H. (2011). Effectiveness of Applying the Strategy (Preview-View-Review) in Developing Direct Literal Reading Comprehension Skills for the Deaf Female Students, Fifth Grade Primary School at Al-amal Institute in the West of Riyadh (Unpublished master's thesis). Riyadh: King Saud University.

Allen, T. E. (2015). ASL Skills, Fingerspelling Ability, Home Communication Context and Early Alphabetic Knowledge of Preschool-Aged Deaf Children. Sign Language Studies, 15, 233-265. https://doi.org/10.1353/sls.2015.0006

Alnagi, S. (2010). Teaching methods of reading prevailing among teachers of the deaf students in primary stage in Riyadh (Unpublished master's thesis). King Saud University, Riyadh.

Berent, G. P. (2001). English for Deaf Students: Assessing and Addressing Learners' Grammar Development. In D. Janáková (Ed.), International Seminar on Teaching English to Deaf and Hard-of-Hearing Students at Secondary and Tertiary Levels of Education: Proceedings (pp. 124-134). Prague, Czech Republic: Charles University, The Karolinum Press.

Emmorey, K., \& Petrich, J. F. (2012). Processing Orthographic Structure: Associations between Print and Fingerspelling. Journal of Deaf Studies and Deaf Education, 17, 194-204. https://doi.org/10.1093/deafed/enr051

Fayad, H. (2004). Develop Some Reading Comprehension Skills for Deaf and Hard of hearing Students in Secondary Level (Unpublished Master's Thesis). Cairo: Ain Shams University.

Gaston, S. H. (2009). Supporting Deaf Students' Development of English Spelling Skills Creating ASL Fingerspelling Stories. (Unpublished Master's Thesis). San Diego, CA: University of California.

Goldin-Meadow, S., \& Mayberry, R. I. (2001). How Do Profoundly Deaf Children Learn to Read? Learning Disabilities Research \& Practice, 16, 222-229. https://doi.org/10.1111/0938-8982.00022

Golos, D. B., \& Moses, A. M. (2013). Developing Preschool Deaf Children's Language and Literacy Learning from an Educational Media Series. American Annals of the Deaf, 158, 
411-425. https://doi.org/10.1353/aad.2013.0039

Grushkin, D. A. (1998). Lexidactylophobia: The (Irrational) Fear of Fingerspelling. American Annals of the Deaf, 143, 404-415. https://doi.org/10.1353/aad.2012.0192

Haptonstall-Nykaza, T. S., \& Schick, B. (2007). The Transition from Fingerspelling to English Print: Facilitating English Decoding. Journal of Deaf Studies and Deaf Education, 12, 172-183. https://doi.org/10.1093/deafed/enm003

Hile, A. (2009). Deaf Children's Acquisition of Novel Fingerspelled Words (Unpublished Doctoral Dissertation). Boulder, CO: University of Colorado.

Humphries, T., \& MacDougall, F. (1999). “Chaining” and Other Links: Making Connections between American Sign Language and English in Two Types of School Settings. Visual Anthropology Review, 15, 84.94. https://doi.org/10.1525/var.2000.15.2.84

Kim, S., Im, H., \& Kwon, K. (2015). The Role of Home Literacy Environment in Toddlerhood in Development of Vocabulary and Decoding Skills. Child \& Youth Care Forum, 44, 835-852. https://doi.org/10.1007/s10566-015-9309-y

Padden, C., \& Ramsey, C. (2000). American Sign Language and Reading Ability in Deaf Children. In C. Chamberlain, J. Morford, \& R. Mayberry (Eds.), Language Acquisition by Eye (pp. 65-90). London: Lawrence Erlbaum.

Prinz, P., \& Strong, M. (1997). A Study of the Relationship between American Sign Language and English Literacy. Journal of Deaf Studies and Deaf Education, 2, 37-46. https://doi.org/10.1093/oxfordjournals.deafed.a014308

Prinz, P. M., \& Strong, M. (1998). ASL Proficiency and English Literacy within a Bilingual Deaf Education Model of Instruction. Topics in Language Disorders, 18, 47-60. https://doi.org/10.1097/00011363-199808000-00006

Puente, A., Alvarado, J., \& Herrera, V. (2006). Fingerspelling and Sign Language as Alternative Codes for Reading and Writing Words for Chilean Deaf Signers. American Annals of the Deaf, 151, 299-310. https://doi.org/10.1353/aad.2006.0039

Quinto-Pozos, D., \& Reynolds, W. (2012). ASL Discourse Strategies: Chaining and Connecting: Explaining across Audiences. Sign Language Studies, 12, 41-65. https://doi.org/10.1353/sls.2011.0021

Roos, C. (2013). Young Deaf Children's Fingerspelling in Learning to Read and Write: An Ethnographic Study in a Signing Setting. Deafness \& Education International, 15, 149-178. https://doi.org/10.1179/1557069X13Y.0000000020

Sarchet, T., Marschark, M., Borgna, G., Convertino, C., Sapere, P., \& Dirmyer, R. (2014). Vocabulary Knowledge of Deaf and Hearing Postsecondary Students. Journal of Postsecondary Education and Disability, 27, 161-178.

Schwartz, L. (2011). Fingerspelling as a Phonological Code for Deaf and Hard of Hearing Students (Unpublished Master's Thesis). Boulder, CO: Colorado University.

Staden, A., \& Roux, N. (2010). The Efficacy of Fingerspell Coding and Visual Imaging Techniques in Improving the Spelling Proficiency of Deaf Signing Elementary-Phase Children: A South African Case Study. Journal of Developmental \& Physical Disabilities, 22, 581-594. https://doi.org/10.1007/s10882-010-9196-y

Stone, A., Kartheiser, G., Hauser, P. C., Petitto, L.-A., \& Allen, T. E. (2015). Fingerspelling as a Novel Gateway into Reading Fluency in Deaf Bilinguals. PLoS ONE, 10, e0139610. https://doi.org/10.1371/journal.pone.0139610

Trussell, J. W., \& Easterbrooks, S. R. (2014). The Effect of Enhanced Storybook Interaction on Signing Deaf Children's Vocabulary. Journal of Deaf Studies \& Deaf Education, 19, 319-332. https://doi.org/10.1093/deafed/ent055 
Visual Language and Visual Learning Science of Learning Center (2010). The Importance of Fingerspelling for Reading (Research Brief No. 1). Washington, DC: Sharon Baker.

Williams, C. (2012). Promoting Vocabulary Learning in Young Children Who Are Deaf and Hard of Hearing: Translating Research into Practice. American Annals of the Deaf, 156, 501-508. https://doi.org/10.1353/aad.2012.1597

Williams, J., Darcy, I., \& Newman, S. (2015). Fingerspelling and Print Processing Similarities in Deaf and Hearing Readers. Journal of Language and Literature, 6, 56-65. 